\title{
Influencia del ciclo lunar en la captura de langosta espinosa Panulirus argus (Decapoda: Palinuridae) en el Golfo de Batabanó, Cuba
}

\author{
Alexander Lopeztegui ${ }^{1}$, Julio A. Baisre ${ }^{2} \&$ Norberto Capetillo $^{1}$ \\ 1. Centro de Investigaciones Pesqueras, 5ta Ave. y 246, Santa Fé, Playa, Ciudad de La Habana, Cuba; \\ sasha@cip.telemar.cu; norberto@cip.telemar.cu \\ 2. Ministerio de la Industria Alimentaria, calle 14 entre 1ra y 3ra, Miramar, Ciudad de la Habana, Cuba; \\ baisre@mip.telemar.cu
}

\author{
Recibido 01-II-2010. Corregido 19-VII-2010. Aceptado 19-VIII-2010.
}

\begin{abstract}
Influence of lunar cycle on catches of spiny lobster Panulirus argus (Decapoda: Palinuridae) in the Gulf of Batabanó, Cuba. Many biological processes such as reproductive and migratory behaviours have been associated with moon cycles. In this study, the nocturnal light levels associated with lunar cycle (INT) were correlated with daily catch rate of lobster P. argus, during seven lunar months of 2002 fishing period, to determine a possible relationship between these variables. The lobster catches were obtained from three fishing companies that develop their activities in the Gulf of Batabanó: EPICOL that fishes in Coloma area; PESCAHABANA in Batabanó area and PESCAISLA in Isla area. Daily catch per boat (CDB) was used as a measurement of daily catch variations (catch rate). The correlation was analyzed showing it in chronological graphs based on average of CDB per lunar phases, comparing lobster catch rate per lunar phases -with the Kruskal-Wallis test-. Spearman rank correlation coefficient and cross correlation techniques were also applied. Similarities between lobster catch rate and the lunar cycle were not found. Spearman rank correlation coefficient was modularly smaller than 0.1 in all cases and demonstrated quantitatively that correlation between CDB and INT does not exist. Kruskal-Wallis test detected differences only in Batabanó area but not when making the analyses for the whole Gulf of Batabanó. Finally, the cross correlations do not detected significance in any zone, as well. It is concluded that, in opposition to what other authors have reported, the catch rates of P. argus and the lunar cycle did not show significant correlation in the Gulf of Batabanó. This trend was independent of the fishing art, which varied according to the time of the year that was analyzed. Rev. Biol. Trop. 59 (1): 207-216. Epub 2011 March 01.
\end{abstract}

Key words: Panulirus argus, spiny lobster, lunar cycle, catch rate, cross correlation, Gulf of Batabanó.

La luna constituye un punto de referencia u orientación para los organismos, que marca el comienzo de determinadas conductas vinculadas fundamentalmente con procesos reproductivos y migratorios. En crustáceos, la abundancia de larvas y sus movimientos, y el proceso de asentamiento, han sido correlacionados con el ciclo lunar, obteniendo los mayores valores de estos parámetros en las fases lunares más oscuras (Kingsford et al. 2002, Criales et al. 2005). La relación entre el ciclo lunar y la conducta de los organismos marinos ha sido utilizada en favor de las pesquerías. La tasa de captura de Melicertus latisulcatus es menor durante luna nueva, comportamiento utilizado para aumentar la rentabilidad de la pesquería al disminuir el esfuerzo pesquero durante ese período (Dixon \& Roberts 2006). Las migraciones verticales de Xiphias gladius en aguas del Golfo de México y el Caribe se encuentran en estrecha relación con las fases lunares, comportamiento que ayuda también a mejorar los rendimientos en la pesquería de esta especie (Loefer et al. 2007). 
En Panulirus argus (Latreille 1804), langosta espinosa que constituye el principal recurso pesquero de Cuba, se ha demostrado que el asentamiento de puérulos aumenta en los períodos lunares más oscuros, siendo más intenso durante la fase de luna nueva, lo cual contribuye a minimizar el riesgo de depredación (Marx \& Herrnking 1986, Dahlgren \& Staine 2006). Sin embargo, pocos estudios se han realizado acerca de la relación entre las capturas de esta especie y el ciclo lunar (Ziegler 2007). En otras especies de langostas también de importancia comercial como P. cygnus en Australia (Srisurichan et al. 2005) y P. japonicus en Japón (Yamakawa et al. 1994) se ha logrado determinar una relación significativa entre las capturas y el ciclo lunar, siendo éstas mayores durante las fases lunares que mayor oscuridad propician. Sobre $P$. argus sólo un trabajo (Kanciruk 1980) se conoce al respecto en el ámbito internacional. En Cuba, Hernández et al. (1989) realizaron en la región del Golfo de Batabanó, el único estudio sobre $P$. argus vinculado con este tema. En tal investigación se hace referencia a una ritmicidad quincenal de las capturas, siendo mayores en las fases de luna nueva y llena, sin embargo, no queda clara la existencia de una relación significativa entre dichas capturas y el ciclo lunar, por lo que no se llegó a ninguna conclusión efectiva para el manejo pesquero.

En virtud de la relación existente entre el ciclo lunar y los recursos pesqueros, importantes recomendaciones de manejo se podrían hacer. Es posible, por ejemplo, aumentar la rentabilidad de las pesquerías aumentando el esfuerzo sólo en aquellos períodos lunares en los que se determine que los recursos son más vulnerables a las artes de pesca. En este caso es muy importante respetar los límites de explotación para no sobrepescar el recurso. Por otro lado, si es requerido, es posible contribuir a la conservación de la población disminuyendo el esfuerzo pesquero en los períodos lunares antes mencionados. Por tales motivos, es objetivo del presente estudio determinar la relación existente entre las capturas de langosta espinosa $P$. argus y el ciclo lunar, en la región del Golfo de Batabanó, principal zona pesquera de Cuba.

\section{MATERIALES Y MÉTODOS}

Sitio de estudio: El Golfo de Batabanó se ubica en la zona Suroccidental de Cuba. Comprende este golfo una extensa región de plataforma submarina $\left(21285 \mathrm{~km}^{2}\right)$ de $6 \mathrm{~m}$ de profundidad promedio. Limita al Norte con la isla de Cuba y se extiende al Sur hasta el comienzo de las aguas oceánicas, marcado usualmente por cadenas de pequeños cayos de mangle y crestas arrecifales. La actividad de pesca de langosta se realiza dentro de esta área, desde los $21^{\circ} 54^{\prime} 3^{\prime \prime} \mathrm{N}-84^{\circ} 02^{\prime} 1^{\prime \prime} \mathrm{W}$ hasta los $21^{\circ} 39^{\prime} 5^{\prime \prime} \mathrm{N}-81^{\circ} 02$ ' 5" W.

Análisis de los datos: Como expresión de la variación diaria de las capturas se utilizó la captura diaria por barco (CDB), tratada además como una "tasa de captura" y expresada en toneladas por día por barco $(\mathrm{t} / \mathrm{d} / \mathrm{b})$. Los datos de captura utilizados provienen de las tres zonas de pesca en que se divide el Golfo de Batabanó (Coloma, Batabanó e Isla), en estas zonas operan las empresas pesqueras EPICOL, PESCAHABANA y PESCAISLA respectivamente. Los tipos de embarcaciones utilizadas, los períodos de campaña y el área efectiva de pesca son similares para estas tres empresas. Para analizar las capturas del área de Coloma, se utilizaron los datos de todas sus subzonas de pesca con excepción de la zona de Arroyos de Mantua, única ubicada al norte. En el caso de Batabanó y de Isla se analizaron todas las subzonas. Los datos del ciclo lunar se obtuvieron de bases de datos de la NOAA que publica estas series para todo el hemisferio Norte. Se le aplicó la corrección de menos cuatro horas a la iniciación de cada fase lunar para ajustar los datos al horario de Cuba específicamente, debido a que estos datos se publican en T.U. (unidades de tiempo universal del Meridiano de Greenwich).

Se consideraron sólo los meses lunares (Nowinszky 2004), cada uno de los cuales inicia con el primer día de LN (día uno) y termina 
correspondientemente con el último día de la fase del CM. Cada mes lunar se nombró por el mismo nombre del mes fiscal en que comienza la fase de LN, es decir, enero lunar comienza en enero fiscal (con la LN) y termina en febrero fiscal (con el respectivo CM). De esta forma y teniendo en cuenta los períodos de veda establecidos para el año 2002, fue posible el análisis de siete meses o ciclos lunares (enero, junio, julio, agosto, septiembre, octubre y noviembre) para cada zona estudiada.

Mediante la prueba de normalidad de Kolmogorov-Smirnov $(\mathrm{p}<0.001)$ se comprobó que los datos no se ajustan a una distribución normal y mediante la prueba de homocedasticidad de Levene se confirmó que tampoco presentan homogeneidad de varianza, por lo que se aplicaron métodos estadísticos no paramétricos. El análisis se realizó, primeramente, agrupando los datos por fases lunares: luna nueva (LN), cuarto creciente (CC), luna llena (LL) y cuarto menguante (CM). Se calculó y graficó la tasa de captura promedio por fase lunar para cada mes, en cada zona de estudio y para todo el Golfo de Batabanó (las tres zonas en conjunto). Se compararon además, mediante la prueba de Kruskal-Wallis (KW), las medianas de CDB correspondientes a cada fase lunar. Seguidamente, se asumió una continuidad diaria en la variación de intensidad luminosa (INT) asociada al ciclo lunar. Gráficos de tipo cronológico fueron realizados para ilustrar el comportamiento de las variables analizadas. Para la construcción de estos gráficos se estandarizaron los datos de ambas variables (CDB y INT) hallando las anomalías según la fórmula:

$$
\frac{\mathrm{X}(i)-\chi}{\mathrm{s}}
$$

donde X(i) es cada valor de la serie de datos, $\chi$ es la media, y $s$ es la desviación estándar. Como parte de esta etapa se realizó el análisis de correlación.

Para la determinación de correlaciones, la variación de intensidad luminosa asociada al ciclo lunar fue analizada asignando valores arbitrarios de INT a los diferentes días del ciclo lunar. Al primer día de la fase LL le fue asignado el valor de uno (máxima intensidad luminosa), y al primer día de la fase $\mathrm{LN}$ el valor de cero (mínima intensidad luminosa). Según esta representación (adimensional), el primer día de las fases $\mathrm{CM}$ y $\mathrm{CC}$ se corresponden con una intensidad luminosa de 0.5 en ambos casos. De esta manera, los períodos lunares más oscuros son $\mathrm{CM}$ y $\mathrm{LN}$, con intensidades luminosas que varían de 0.5 a 0 y de 0 a 0.5 respectivamente $(0 \leq \mathrm{INT} \leq 1)$. Las correlaciones se realizaron entre los valores de INT asignados y los valores calculados de CDB. Se determinó en primer lugar el coeficiente de correlación por rangos de Spearman (R), el cual se presenta conjuntamente con los gráficos cronológicos según la zona analizada en cada caso. Además se aplicó la técnica de correlación cruzada, la cual permite obtener un coeficiente que ha sido utilizado por varios autores en la determinación de la relación que guardan las tasas de captura de langosta con el ciclo lunar (Acosta et al. 1997, Srisurichan et al. 2005).

Para el análisis de correlación cruzada se transformaron lo datos de CDB según la función de autocorrelación $x=x-\left(a+b^{*} x\right.$ (lag)) aplicada para un retardo (lag) de +1 , dado que para este retardo fueron encontradas las mayores autocorrelaciones en las series de datos. $\mathrm{La}$ función de autocorrelación permite eliminar de las series originales las tendencias estacionales y las autocorrelaciones y correlaciones parciales que puedan existir, por lo que las series pueden ser tratadas como procesos de "ruido blanco". Mediante el modelo ARIMA, provisto por el programa Statistica 6.0 y basado en los criterios de Box \& Jenkins (1976), se comprobó que ninguna otra transformación era precisa para ajustar las series de datos. La verificación de que las series ya transformadas presentan un comportamiento similar a un proceso de "ruido blanco" se realizó mediante el estadístico Q (Ljung \& Box 1978). Mediante la técnica de promedios móviles (moving averages, q-Q) se confirmó que las tendencias estacionales (presentes en las series antes de ser transformadas) lograron ser removidas. Para la realización de los análisis de correlaciones cruzadas se trabajó con un retardo máximo de 14 , asumiendo que 
la duración media de cada ciclo lunar es aproximadamente 29.53 días (Strahler 1975). Tanto los análisis de correlaciones cruzadas, como las comparaciones de medianas y los coeficientes de correlación, se calcularon para una significación de $\mathrm{p}<0.05$.

\section{RESULTADOS}

Al graficar los promedios mensuales de CDB por fase lunar, se aprecia que no es posible determinar un patrón en la distribución de los valores. La CDB promedio en la fase de CM correspondiente al mes de enero, por ejemplo, puede ser tanto la más alta en Isla, como la más baja en Batabanó. De modo similar, el promedio de CDB en CC para la zona de Coloma, por ejemplo, puede ser lo mismo el más alto en julio, como el más bajo en septiembre. No puede expresarse por tanto, que durante alguna fase lunar las tasas de captura se comportan regularmente bajas, o altas. Este comportamiento se verifica para cada zona y al analizar el Golfo de Batabanó como una sola región (Fig. 1).

Los gráficos cronológicos realizados para ilustrar posibles similitudes en los valores de las variables analizadas (CDB e INT), muestran que existen diferencias en la oscilación de dichos valores. Un patrón bien definido y regular en el tiempo puede observarse para INT, las series de CDB sin embargo no muestran una tendencia similar en ninguna de las zonas. Los coeficientes de correlación calculados (Spearman, $\mathrm{p}<0.05$ ) demuestran la baja correlación existente entre las tasas de captura y la variación de intensidad luminosa asociada al ciclo lunar, en ninguna de las zonas estudiadas este coeficiente resultó significativo (Fig. 2).

La comparación estadística entre las series de $\mathrm{CDB}$ por fase lunar $(\mathrm{KW}, \mathrm{p}<0.05)$ indica que sólo en Batabanó existen diferencias significativas entre las tasas de captura por fase lunar.
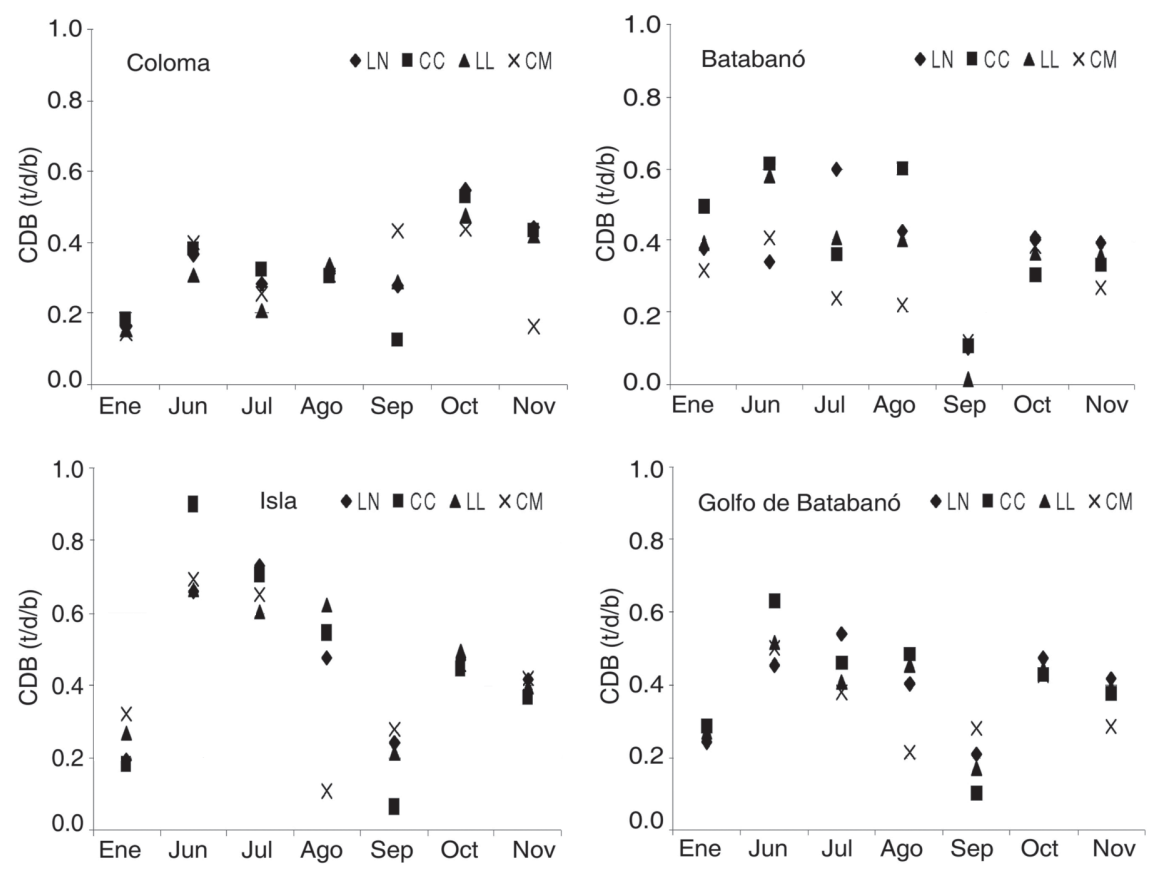

Fig. 1. Promedio mensual de CDB por fase lunar para cada zona de estudio y todo el Golfo de Batabanó (las tres zonas en conjunto).

Fig. 1. Monthly average of CDB per lunar phase for each study zone and for the Gulf of Batabanó (the three zones altogether). 

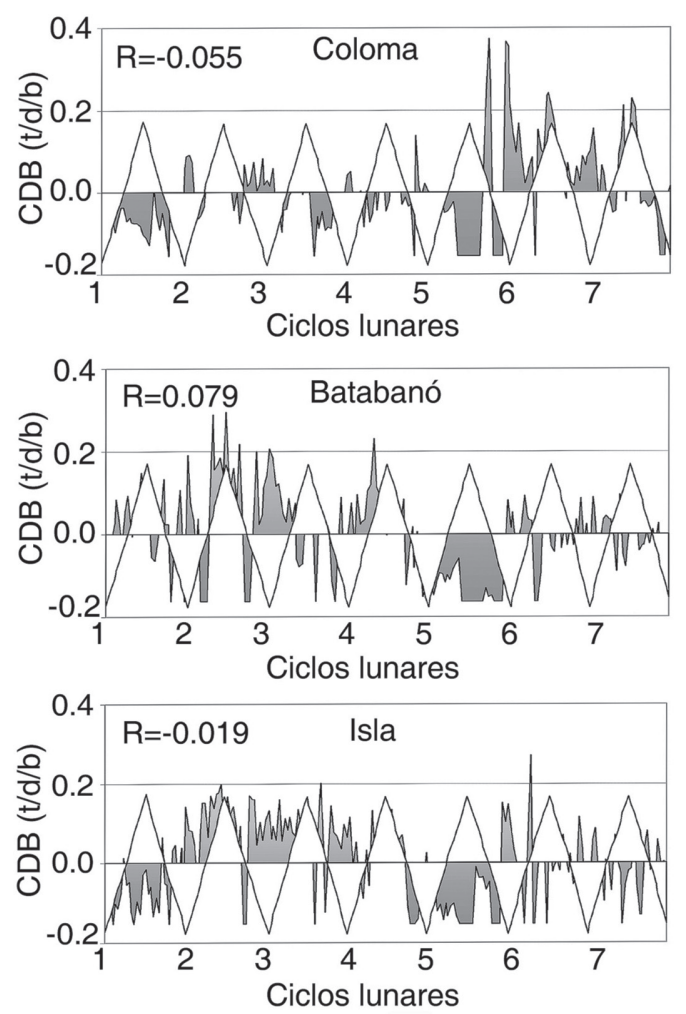

Anomalías CDB $\square$ Anomalías INT

Fig. 2. Gráfico cronológico que ilustra las diferencias entre las anomalías de ambas variables (CDB e INT). R:coeficiente de correlación por rangos de Spearman; *:coeficiente significativo.

Fig. 2. Chronologic graph showing difference between anomalies of both variables (CDB and INT). R:Spearman rank correlation coefficient, *:significant coefficient.

Sin embargo, estas diferencias no se aprecian al integrar los datos de las tres zonas y tratar el Golfo de Batabanó como una sola región de pesca (Fig. 3).

El análisis de correlaciones cruzadas demuestra que para ninguna de las tres zonas se verifican correlaciones significativas entre las tasas de captura y la variación de intensidad luminosa asociada al ciclo lunar. Los coeficientes de correlación cruzada son modularmente inferiores a 0.1 en todos los casos y el patrón de correlación no se repite entre las zonas, lo que demuestra la poca asociación entre el ciclo lunar y las tasas de captura (Fig. 4).

\section{DISCUSIÓN}

No fue posible detectar una relación significativa entre el ciclo lunar y las capturas de $P$. argus mediante ninguno de los procedimientos estadísticos empleados. Todas las técnicas utilizadas demuestran una baja asociación entre las variables CDB e INT. Los coeficientes de correlación calculados (Spearman y correlación cruzada) no resultaron en ninguna zona significativos y presentan ambos valores inferiores a 0.1 (valor modular), lo cual corrobora la existencia de correlaciones estadísticamente no significativas entre el ciclo lunar y la tasa de captura de langostas en la región del Golfo de Batabanó.

Los meses registrados durante este estudio comprenden el período de levante de veda (junio) y los meses de verano (julio y agosto) en que las capturas se comportan con relativamente mayor estabilidad y los datos presentan en consecuencia mayor regularidad. En estos meses el arte de pesca con más frecuencia utilizado en la zona de estudio es el pesquero de fondo o pesquero tradicional. Los meses de invierno (octubre y noviembre), comprenden la época de recalo o de migraciones otoñales, período en que se hace más frecuente el empleo del jaulón y la nasa como artes de pesca, aunque no deja de utilizarse totalmente el pesquero de fondo. El hecho de no haber encontrado correlaciones significativas entre las tasas de captura de $P$. argus y el ciclo lunar, aún habiéndose considerado épocas (verano e invierno) con regímenes ambientales diferentes, e incluso con diferencias en el arte de pesca con más frecuencia utilizado, hace posible plantear que esta relación no significativa entre las capturas y el ciclo lunar es independientemente del arte de pesca o la época del año que se analice.

El hecho de no haber encontrado una correlación significativa entre las capturas de $P$. argus y el ciclo lunar en la zona del Golfo de Batabanó, no se contradice en ninguna medida con lo planteado por otros autores. Por un lado, puede ser que $P$. argus en aguas de la plataforma cubana, tenga un comportamiento similar al que varios autores reportan para 

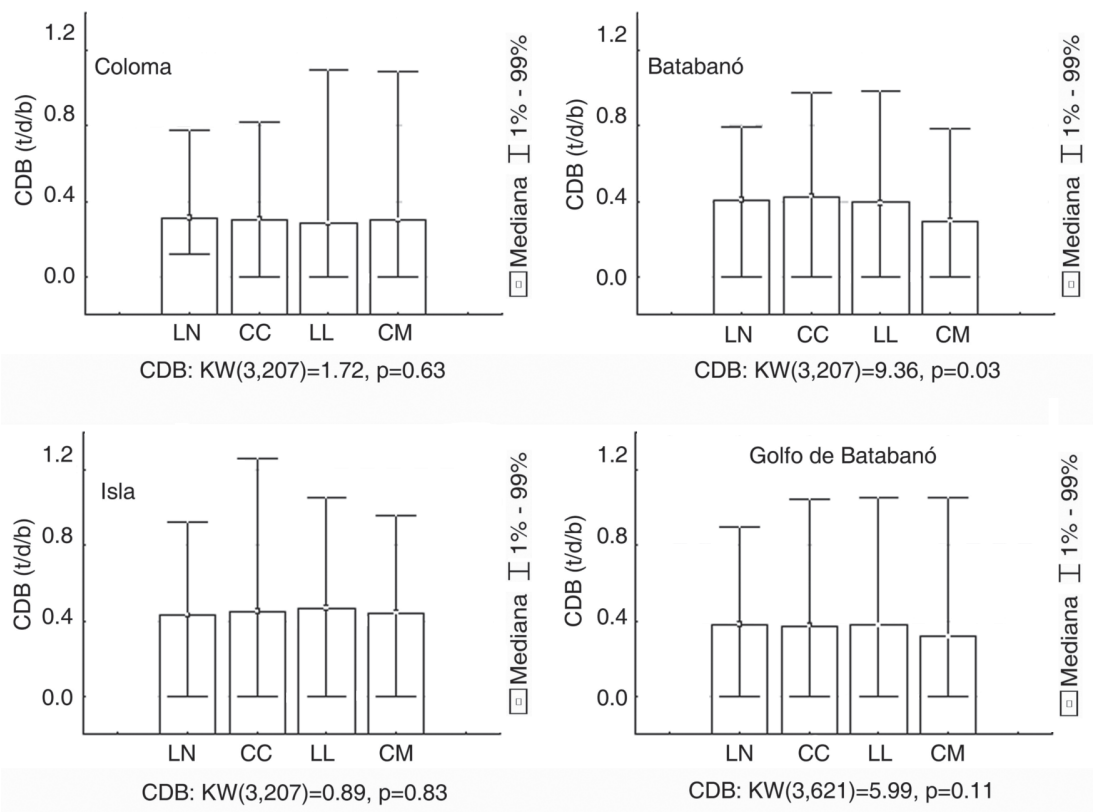

Fig. 3. Tasas de captura por fase lunar en las tres zonas muestreadas y para todo el Golfo de Batabanó.

Fig. 3. Catch rates per lunar phases for the three sampled zones and for the Gulf of Batabanó.

algunas especies del género Jasus (Booth 1989, Keulder 2005), en las que se ha comprobado una independencia entre las capturas y las fases lunares.

Sobre algunas especies del género Рапиlirus, incluso sobre la propia especie $P$. argus, varios autores a nivel mundial han encontrado correlaciones significativas entre las tasas de captura y el ciclo lunar (Morgan 1974, Kanciruk 1980, Srisurichan et al. 2005). Sin embargo, esto no significa que tales correlaciones tengan necesariamente que existir para $P$. argus en aguas de la plataforma cubana, pues las poblaciones de distintas regiones geográficas están sometidas a condiciones ambientales diferentes y a las particularidades del sistema de manejo pesquero imperante en cada área o país. Afectaciones meteorológicas como huracanes y frentes fríos, e incluso el sólo aumento en la fuerza de los vientos, pueden regir el comportamiento de las capturas de langosta más que el ciclo lunar (Hernández et al. 1989). Kingsford et al.
(2002) plantean que la influencia de los ciclos lunares sobre la conducta de los organismos tiene límites de distancia de aproximadamente $100 \mathrm{~km}$. Es decir, poblaciones que se encuentren separadas $100 \mathrm{~km}$ o más, pueden potencialmente ser influenciadas de forma diferente por el ciclo lunar. Lowry et al. (2007) argumentan que la determinación de una relación entre las tasas de captura de cualquier especie y el ciclo lunar, está indiscutiblemente condicionada a las peculiaridades del sistema pesquero de la región para la que se realice el análisis.

Respecto a lo planteado con anterioridad específicamente en Cuba, Hernández et al. (1989) reportan para la zona del Golfo de Batabanó, que las capturas de langosta presentan una ligera tendencia a ser mayores en las fases de LN y LL, sin embargo, en este caso no se demostró estadísticamente la existencia de correlaciones significativas. Por tal motivo, puede plantearse que los resultados obtenidos por tales autores no se contradicen con los 

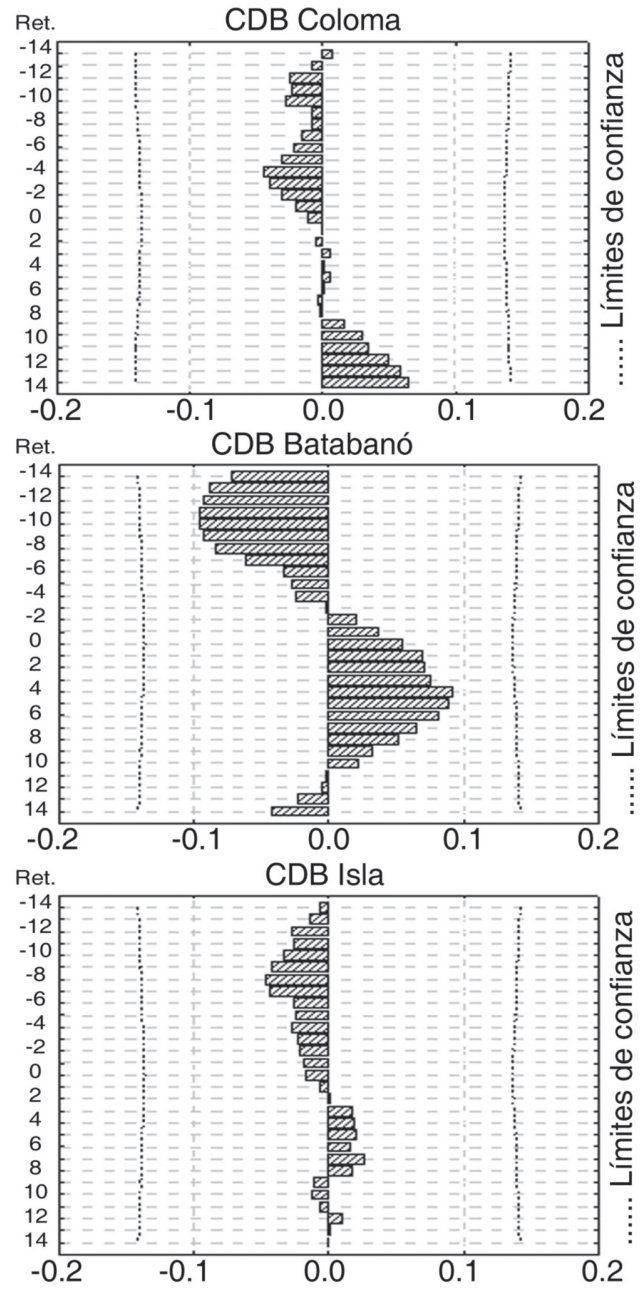

Fig. 4. Correlación cruzada entre CDB (variable retardada) e INT (variable fija) para cada zona estudiada del Golfo de Batabanó. Eje $x$ :valor del coeficiente de correlación cruzada; Ret.:retardo.

Fig. 4. Cross correlation between CDB (lagged variable) and INT (fix variable) for each study zone of the Gulf of Batabanó. Axis $x$ :cross correlation coefficient; Ret:lag.

resultados del presente estudio. Las diferencias en el diseño general de la investigación, y específicamente en el procesamiento estadístico empleado, hacen que ambos trabajos no sean comparables. Son justamente esas diferencias en cuanto al procesamiento estadístico las que sugieren mayor confiabilidad a los resultados que actualmente se presentan. Además, algunos aspectos de carácter ecológico en relación a los cambios de conducta que experimentan las langostas a consecuencia del ciclo lunar, no apoyan los resultados obtenidos por Hernández et al. (1989). Cuando la pesquería se realiza con nasas o jaulones, las capturas aumentan en períodos lunares oscuros (CM-LN) debido a que las langostas aumentan la frecuencia y amplitud de sus búsquedas de alimento, pues la oscuridad hace que disminuya el riesgo de que sean depredadas (Srisurichan et al. 2005), no se explica entonces que, como plantearon Hernández et al. (1989), las capturas aumenten también en períodos lunares de alta iluminación (LL).

Debido a la falta de estandarización en los procedimientos estadísticos comúnmente utilizados para abordar estudios de correlación entre el ciclo lunar y variables de origen diverso, una complementación entre varias técnicas es recomendada para la obtención de resultados confiables (DeBruyn \& Meeuwig 2001). El procesamiento de los datos y los gráficos presentados en este estudio para ilustrar la falta de correlación entre las capturas y el ciclo lunar, han sido descritos y utilizados por varios investigadores a nivel mundial. Entre los métodos más comunes para realizar análisis de esta naturaleza, están los que hacen un tratamiento por categorías de los datos subdividiendo en cuatro fases (LN, CC, LL y CM) el ciclo lunar. El test de Kruskal-Wallis, se puede citar entre las metodologías más empleadas en este caso (Von Herbing \& Hunte 1991). Otro grupo de autores han realizado sus estudios asumiendo una distribución continua (diaria) de los datos del ciclo lunar, el coeficiente de correlación por rangos de Spearman es frecuentemente utilizado en estos casos (Oxenford et al. 1995). Un tercer grupo de investigadores han desechado los análisis estadísticos complejos y han empleando simples representaciones gráficas para demostrar la periodicidad lunar y su relación con la conducta de diversos organismos (Dufour et al. 1996).

Los análisis de series de tiempo, y especialmente las correlaciones cruzadas, son en 
la actualidad uno de los procedimientos más frecuentemente utilizados para analizar la relación de las tasas de captura de diferentes recursos pesqueros, incluyendo varias especies de langostas, con el ciclo lunar (Srisurichan et al. 2005, Ziegler 2007, Gatica et al. 2009). A pesar de ser una técnica bien avalada y en constante perfeccionamiento por varios autores de la comunidad científica internacional (Podobnik \& Stanley 2008, Arianos \& Carbone 2009), en Cuba su uso no ha sido aún muy difundido. El empleo de correlaciones cruzadas en investigaciones de carácter ambiental, y particularmente en estudios ecológicos relacionados con los recursos pesqueros, podría elevar el rigor de las investigaciones realizadas y contribuir así a generar mejores respuestas ante las problemáticas planteadas.

La existencia de correlaciones no significativas entre el ciclo lunar y la tasa de captura de $P$. argus en el Golfo de Batabanó, tiene implicaciones importantes para el manejo pesquero del recurso, ya que se pueden planificar las campañas de pesca y administrar el esfuerzo pesquero, con total independencia respecto a las fases lunares. La variación de intensidad luminosa asociada al ciclo lunar, que en otras regiones del mundo debe tenerse en cuenta en la planificación de las actividades de pesca, en Cuba no influye significativamente sobre las capturas, por lo que puede plantearse que las langostas son igualmente vulnerables a la pesquería en cualquier fase o día del ciclo lunar. De acuerdo con las características del sistema de ordenamiento pesquero vigente en Cuba, las variaciones diarias en la tasa de captura de langosta en la zona del Golfo de Batabanó, no se explican en ninguna medida por la variación de iluminación nocturna asociada al ciclo lunar.

El hecho de no haberse encontrado una correlación significativa entre las capturas de langosta y el ciclo lunar no implica que dicho ciclo definitivamente no influya en la conducta de las langostas. Por un lado, puede ser posible que, si existen variaciones en la conducta de $P$. argus como consecuencia de variaciones en los niveles de iluminación nocturna asociados al ciclo lunar, éstas no sean significativas como para reflejarse en las capturas. Por otro lado puede ser que, la influencia de la luna sobre la conducta de las langostas no sea tan marcada en cuanto al efecto de la luz y sí en cuanto al efecto gravitacional, en cuyo caso se debería estudiar la relación entre el ciclo de mareas y las capturas. Nuevas investigaciones, con otro diseño experimental y posiblemente en la meso-escala, se requerirían para comprobar lo anteriormente planteado. Teniendo en cuenta que la influencia de la luna sobre las poblaciones de organismos puede variar de una región geográfica a otra, y que para poblaciones de $P$. argus residentes en otros países tropicales se ha encontrado una relación significativa entre el ciclo lunar y las capturas, pudiera ser factible la realización de estudios similares en el resto de las regiones de pesca de Cuba, y si es posible, ampliar la escala temporal de las investigaciones a fin de tener en cuenta la variabilidad interanual de fenómenos ambientales fundamentalmente.

\section{AGRADECIMIENTOS}

A los investigadores María Estela de León y Rafael Puga por los señalamientos realizados durante el diseño y ejecución de la investigación. A Anna Carbone por sus trabajos en el tema de correlaciones cruzadas. Muy especialmente a Beatriz Martínez Daranas por la revisión general de este documento.

\section{RESUMEN}

Muchos procesos biológicos tales como los comportamientos reproductivos y migratorios se han asociado con los ciclos de la luna. Con el objetivo de determinar la relación entre las capturas de langosta espinosa Panulirus argus (Decapoda: Palinuridae) y el ciclo lunar, en la zona del Golfo de Batabanó, se correlacionaron los niveles de iluminación nocturna del ciclo lunar (INT) con la captura diaria por barco (CDB) durante siete meses lunares correspondientes al período de pesca del 2002. Los datos de captura provinieron de las tres empresas pesqueras que realizan su actividad en la zona del Golfo de Batabanó. La relación entre el ciclo lunar y las capturas de langostas fue examinada mediante varios procedimientos estadísticos. Los primeros gráficos realizados, cualitativos, 
mostraron que no existe relación entre las tasas de captura y el ciclo lunar. Los coeficientes de Spearman calculados, modularmente menores que 0.1 en todos los casos, demostraron cuantitativamente que no existe correlación entre las mencionadas variables. La prueba de Kruskal-Wallis detectó diferencias sólo en una de las tres áreas estudiadas. Las correlaciones cruzadas no detectaron significación en ninguna de las zonas, con coeficientes de correlación cruzada modularmente inferiores a 0.1 . A diferencia de lo reportado por otros autores, las capturas de P. argus y el ciclo lunar no presentan correlación significativa en el Golfo de Batabanó.

Palabras clave: Panulirus argus, langosta espinosa, ciclo lunar, tasa de captura, correlación cruzada, Golfo de Batabanó

\section{REFERENCIAS}

Acosta, C.A., T.R. Matthews \& M.J. Butler. 1997. Temporal patterns and transport processes in recruitment of spiny lobster (Panulirus argus) postlarvae to south Florida. Mar. Biol. 129: 79-85.

Arianos, S. \& A. Carbone. 2009. Cross-Correlation of long-range correlated series. J. Stat. Mech. (también disponible en línea: http://iopscience.iop. org/1742-5468/2009/03/P03037/pdf.

Booth, J.D. 1989. Occurrence of the puerulus stage of the rock lobster, Jasus edwardsii at the New Plymouth Power Station, N.Z. J. Mar. Freshw. Res. 23: 43-50.

Box, G. \& G. Jenkins. 1976. Time series analysis: forecasting and control. Holden Day, Oakland, California, EEUU.

Criales, M.M., J.D. Wang, J.A. Browder, M.B. Robblee, T.L. Jackson \& C. Hittle. 2005. Variability in supply and cross-shelf transport of pink shrimp (Farfantepenaeus duorarum) postlarvae into western Florida Bay. Fish. Bull. 104: 60-74.

Dahlgren, C.P. \& F. Staine. 2006. Spatial and temporal patterns of caribbean spiny lobster, Panulirus argus, puerulus settlement to the belizean lagoon system. Book of Abstracts of the $59^{\text {th }}$ Annual Program of Gulf and Caribbean Fisheries Institute, Belize. (también disponible en línea: www.gcfi.org/Conferences/59th /59BookofAbstracts.pdf).

DeBruyn, A.M.H. \& J.J. Meeuwig. 2001. Detecting lunar cycles in marine ecology: periodic regression versus categorical ANOVA. Mar. Ecol. Prog. Ser. 214: 307-310.
Dixon, C.D. \& S.D. Roberts. 2006. West Coast Prawn (Melicertus latisulcatus) Fishery. Fishery Assessment Report to PIRSA Fisheries. SARDI Aquatic Sciences Publication. Research Report Series, Australia.

Dufour, V., E. Riclet \& A. Lo-Yat. 1996. Colonization of reef fishes at Moorea Island, French Polynesia: temporal and spatial variation of the larval flux. Mar. Freshw. Res. 47: 413-422.

Gatica, C., R.A. Quiñones, D. Figueroa, R. Wiff, E. Navarro \& M. Donoso. 2009. Asociación entre la Corriente de Deriva de los vientos del Oeste y la abundancia relativa del pez espada (Xiphias gladius) frente a la costa de Chile. Lat. Am. J. Aquat. Res. 37: 97-105.

Hernández, B., C. García \& P. Sierra. 1989. Algunos aspectos de las fases lunares y su relación con las capturas de langosta Panulirus argus en el Golfo de Batabanó. Rev. Cub. Invest. Pes.14: 72-89.

Kanciruk, P. 1980. Ecology of juvenile and adult Palinuridae, p. 59-96. In J.S. Cobb \& B.F. Phillips (eds.). The biology and management of lobsters. Vol. II. Ecology and Management. Academic Press, Nueva York, EEUU.

Keulder, F.J. 2005. Puerulusand early juvenile recruitment of the rock lobster Jasus lalandii in relation to the environment at Luderitz Bay, Namibia. MSc. Thesis, Rhodes University, Namibia.

Kingsford, M.J., M. Jeffrey, A.L. Shanks, K.C. Lindeman, S.G. Morgan \& J. Pineda. 2002. Sensory environments, larval abilities and local self-recruitment. Bull. Mar. Sci. 70: 309-340.

Ljung, G.M. \& G.E.P. Box. 1978. On a measure of lack of fit in time series models. Biometrica 65: 297-303.

Loefer, J., G.R. Sedberry \& J.C. McGovern. 2007. Nocturnal depth distribution of western North Atlantic swordfish (Xiphias gladius, Linnaeus, 1758) in relation to lunar illumination. Gulf and Caribbean Res. 19: $83-88$.

Lowry, M., D. Williams \& Y. Metti. 2007. Lunar-landingsrelationship between lunar phases and catch rates for an Australian gamefish-tournament fishery. Fish. Res. 88: $15-23$.

Marx, J.M. \& W.F. Herrnking. 1986. Species Profiles: Life Histories and Environmental Requirements of Coastal Fishes and Invertebrates (South Florida), spiny lobster. U.S. Fish Wildl. Serv. Biol. Rep. 82: 4-21.

Morgan, G.R. 1974. Aspects of the population dynamics of the western rock lobster, Pulirus cygnus George. II 
Seasonal changes in the catchability coefficient. Aust. J. Mar. Freshw. Res. 25: 249-259.

Nowinszky, L. 2004. Nocturnal Illumination and Night Flying Insects. Applied Ecol. and Environ. Res. 2: $17-52$.

Oxenford, H.A., R. Mahon \& W. Hunte. 1995. Distribution and relative abundance of flyingfish (Exocoetidae) in the eastern Caribbean. III. Juveniles. Mar. Ecol. Prog. Ser. 117: 39-47.

Podobnik, B. \& H.E. Stanley. 2008. Detrended CrossCorrelation Analysis: A New Methods for Analyzing two Nonstationary Time Series. Phys. Rev. Lett. 100. 084102: 1-4.

Srisurichan, S., N. Caputi \& J. Cross. 2005. Impact of lunar cycle and swell on the daily catch rate of western rock lobster (Panulirus cygnus) using time series modelling. N.Z. J. Mar. Freshw. Res. 39: 749-764.

Strahler, A.1975. Geografía Física. Omega S.A. Barcelona, España.

Von Herbing, I.H. \& W. Hunte. 1991. Spawning and recruitment of the bluehead wrasse Thalassoma bifasciatum in Barbados, West Indies. Mar. Ecol. Prog. Ser. 72: 49-58.

Yamakawa, T., Y. Matsumiya, M. Nishimura \& S. Ohnishi. 1994. Expanded DeLury's method with variable catchability and its application to catch-effort data from spiny lobster fishery. Fish. Sci. 60: 59-63.

Ziegler, T.A. 2007. Larval release rhythms and larval behaviour of Palinurid lobsters: a comparative study. Ph.D. Thesis, Duke University, Durham, North Carolina, EEUU. 